\title{
Background subtraction for the Cluster/CODIF plasma ion mass spectrometer
}

\author{
C. G. Mouikis, L. M. Kistler, G. Wang, and Y. Liu \\ Space Science Center, University of New Hampshire, Durham, New Hampshire, USA \\ Correspondence to: C. G. Mouikis (chris.mouikis@unh.edu) \\ Received: 18 April 2013 - Published in Geosci. Instrum. Method. Data Syst. Discuss.: 27 September 2013 \\ Revised: 17 February 2014 - Accepted: 3 March 2014 - Published: 16 April 2014
}

\begin{abstract}
The CODIF instrument on the Cluster spacecraft is a time-of-flight (TOF) ion mass spectrometer. Although TOF spectrometers are relatively immune to background contamination due to the inherent double coincidence requirement, high background rates can still result in false coincidences. Along the Cluster orbit, false coincidences are commonly observed due to the penetrating radiation of relativistic electrons during the encounters with the Earth's radiation belts. A second type of background in these instruments occurs when events of one species fall into the time-of-flight range defined for another species. Although the fraction of the $\mathrm{H}^{+}$events that spill into the $\mathrm{He}^{+}$measurement is small, when the actual $\mathrm{He}^{+}$fluxes are low this can result in significant contamination. In this paper we present two techniques that allow the subtraction of the false coincidences and the $\mathrm{H}^{+}$"spill" from the CODIF measurements.
\end{abstract}

\section{Introduction}

Spaceborne time-of-flight (TOF) ion mass spectrometers are inherently less susceptible to background noise than all-ion instruments (Wang et al., 2011) because of the coincidence requirements for a measurement to be registered as a valid event (Wüest, 1998; Kistler et al., 1999). However, when the background rate is high, false coincidences lead to contamination of the measurements. Along the orbit of the Cluster spacecraft (Escoubet et al., 2001), the main source of background contamination is the penetrating relativistic electron radiation of the radiation belts in the inner magnetosphere region. A more rare source of penetrating particles, albeit with similar effects on the instrument contamination, is the solar energetic proton events (SEPs) that are observed during unusually strong solar flare events.

The Cluster ion spectrometry (CIS) experiment onboard the Cluster mission is a two instrument suite designed to study the dynamics of ions inside and in the vicinity of the Earth's magnetosphere (Rème et al., 2001). The experiment consists of the COmposition and DIstribution Function analyzer (CODIF) instrument that measures the full three dimensional ion distribution of the major magnetospheric ions $\left(\mathrm{H}^{+}\right.$, $\mathrm{He}^{++}, \mathrm{He}^{+}$and $\mathrm{O}^{+}$) over the energy range $\sim 40 \mathrm{eV} \mathrm{e}^{-1}$ $40 \mathrm{keV} \mathrm{e}^{-1}$ (Möbius et al., 1998) and the Hot Ion Analyzer (HIA) instrument that provides an all-ion measurement over a similar energy range. The HIA instrument is an electrostatic analyzer (ESA) while the CODIF instrument is a combination of a top-hat ESA followed by a $15 \mathrm{keV}$ post-acceleration and a TOF measurement.

To illustrate the effect of the penetrating radiation, Fig. 1 shows an example of a Cluster encounter with the outer radiation belt as observed by HIA (top panel), the CODIF $\mathrm{H}^{+}$ channel (middle panel) and the CODIF $\mathrm{O}^{+}$channel (bottom panel). Because there is no energy selection for the penetrating radiation, as there is for ions that come through the electrostatic analyzer, the penetrating radiation results in the same count rate at all energies. If the assumed detection efficiencies do not vary significantly with energy, then the calculated energy flux will also be energy independent. Such a contamination signature is clearly seen in the HIA measurements between the black vertical lines (top panel). The temporal variation of the contamination flux levels is due to the spacecraft traversal through the radiation belt. While the HIA measurement is dominated by the penetrating radiation, the CODIF $\mathrm{H}^{+}$measurement is very little affected by it, allowing the identification of energy dependent variations. 


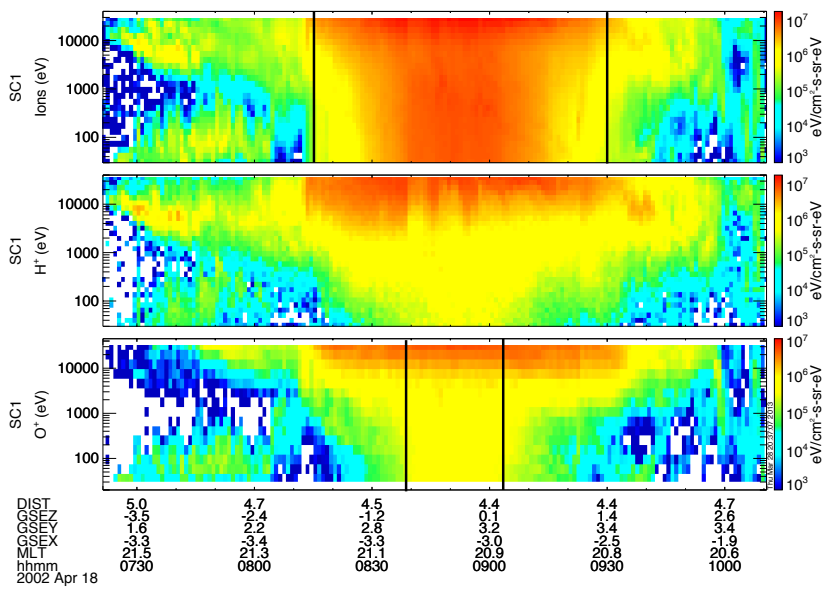

Fig. 1. Cluster encounter with the outer radiation belt as observed by HIA (top panel), CODIF $\mathrm{H}^{+}$channel (middle panel) and CODIF $\mathrm{O}^{+}$channel (bottom panel). The vertical lines indicate the intervals of high contamination for the particular measurement.

The CODIF $\mathrm{O}^{+}$measurement is contaminated, although for a shorter period than HIA, closer to the peak of the radiation fluxes, as indicated by the black vertical lines (bottom panel). However, in this case there is also foreground $\mathrm{O}^{+} \mathrm{ev}-$ ident at energies above $\sim 5 \mathrm{keV}$. Therefore, while the TOF measurement is less susceptible to penetrating radiation contamination, it does not provide total immunity, in particular for the heavier species.

In the following section, we describe the fundamental time-of-flight measurement. In Sect. 3 we present a method to subtract the background from penetrating radiation from the CODIF data. In Sect. 4, we present another background subtraction technique that is used to subtract the fraction of the $\mathrm{H}^{+}$events that "spill" into the $\mathrm{He}^{+}$measurement.

\section{Time-of-Flight measurement}

A significant advantage of the TOF sensors is the inherent coincidence requirement in the TOF measurement which reduces significantly the background contamination, as is demonstrated in Fig. 1. However, for TOF sensors, the foreground to background ratio is energy and species dependent; i.e. assuming the same foreground fluxes for all energies and all species, particles with higher energy and lighter mass will have lower contamination levels.

Figure 2 shows an example (using preflight calibration data) of CODIF time-of-flight spectra for the four major ion species $\mathrm{H}^{+}, \mathrm{He}^{++}, \mathrm{He}^{+}$and $\mathrm{O}^{+}$, at two representative energies, $40 \mathrm{keV} \mathrm{e}^{-1}$ (top panel) and $5 \mathrm{keV} \mathrm{e}^{-1}$ (bottom panel). The ion peaks appear as skewed Gaussian distributions. The tail towards longer times of flight is due to energy loss and scattering in the carbon foil. Time-of-flight windows for each species are defined so that the majority of the TOF distribution for a particular species falls within the correspond-

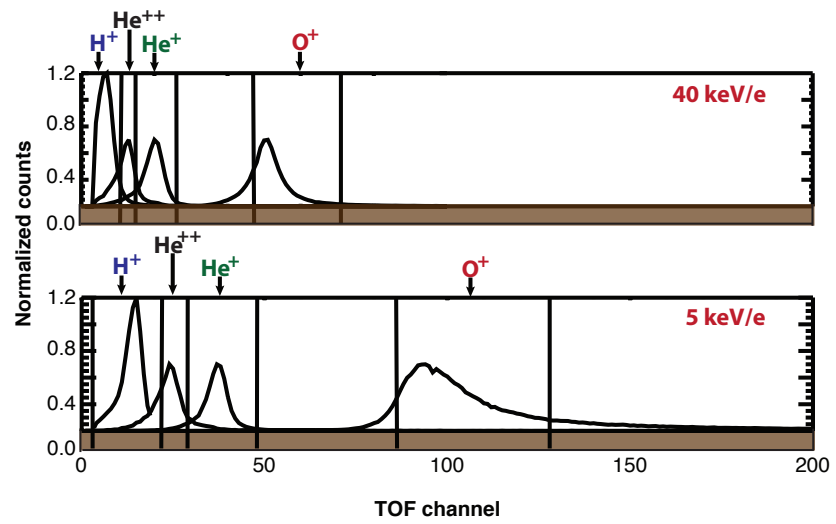

Fig. 2. CODIF time-of-flight (TOF) spectra for two representative energies, 40 and $5 \mathrm{keV} \mathrm{e}^{-1}$. The vertical lines indicate the TOF windows for the four ion species, $\mathrm{H}^{+}, \mathrm{He}^{++}, \mathrm{He}^{+}$and $\mathrm{O}^{+}$.

Table 1. Spillover between species for 40 and $5 \mathrm{keV} \mathrm{e}^{-1}$ particles from S/C 4 CODIF high-sensitivity side pre-flight calibrations.

\begin{tabular}{cccccc}
\hline Energy & Species & \multicolumn{4}{c}{ Spillover fraction in } \\
$\left(\mathrm{keV} \mathrm{e}^{-1}\right)$ & & $\mathrm{H}^{+}$ & $\mathrm{He}^{++}$ & $\mathrm{He}^{+}$ & $\mathrm{O}^{+}$ \\
\hline 40 & $\mathrm{H}^{+}$ & & 0.051 & 0.017 & 0.003 \\
5 & $\mathrm{H}^{+}$ & & 0.011 & 0.003 & 0.001 \\
40 & $\mathrm{He}^{++}$ & 0.286 & & 0.165 & 0.009 \\
5 & $\mathrm{He}^{++}$ & 0.267 & & 0.122 & 0.003 \\
40 & $\mathrm{He}^{+}$ & 0.041 & 0.080 & & 0.002 \\
5 & $\mathrm{He}^{+}$ & 0.005 & 0.041 & & 0.003 \\
40 & $\mathrm{O}^{+}$ & 0.001 & 0.001 & 0.001 & \\
40 & $\mathrm{O}^{+}$ & 0.002 & 0.0 & 0.002 & \\
\hline
\end{tabular}

ing window. The start and end time of the TOF window for each species is marked with vertical lines. The position and width of these TOF windows depends on the particle energy and mass; they are bunched closer together for the high energies and spread out for the lower energies. For both examples shown in Fig. 2, $\mathrm{H}^{+}, \mathrm{He}^{+}$and $\mathrm{O}^{+}$are easily separated while the $\mathrm{He}^{++}$is in the tail of the $\mathrm{H}^{+}$distribution. In fact, the tails of the distribution of each species "spill" into the other species at a certain level. Table 1 shows the spillover fraction of the different species, for the two energies shown in Fig. 2, from pre-flight calibration data obtained from the high-sensitivity side of S/C4. In order to assess the significance of the contamination level between species, the spillover fraction as well as the relative species abundance has to be considered. For example, inside the magnetosphere the $\mathrm{He}^{++}$is almost irretrievable because the spillover from $\mathrm{H}^{+}(\sim 1.1-5.1 \%)$ is large compared to the real $\mathrm{He}^{++}$signal. In contrast, the $\mathrm{H}^{+}$spillover into the $\mathrm{O}^{+}$TOF window is much lower $(\sim 0.1-0.3 \%)$ while the real $\mathrm{O}^{+}$counts are much higher inside the magnetosphere, which results in a much lower contamination level. 
Since the background counts from penetrating radiation are uncorrelated, the background coincidence counts in the time-of-flight spectrum will appear with approximately equal probability in any time-of-flight bin (in Sect. 3.3 we will explain that this is not entirely true) and any energy. Hence, in a TOF spectrum the background shows as a "pedestal" (brown stripe in Fig. 2) on top of which the foreground spectrum resides. However, the number of background counts that will be erroneously characterized as a particular species depends on the width of the TOF window. Therefore, because the TOF window for $\mathrm{H}^{+}$is smaller than the TOF window for $\mathrm{O}^{+}$, there will be fewer erroneous counts in the $\mathrm{H}^{+}$channel than the $\mathrm{O}^{+}$channel. Similarly, since the higher energies have smaller TOF windows than the low energies, there will be fewer erroneous counts in the higher energies than the lower energies.

In conclusion, in a TOF measurement, for a particular background level, the foreground to background ratio is energy and mass dependent and if the ratio is high enough, the background subtraction will result in a statistically significant measurement of the foreground.

\section{Penetrating radiation background subtraction}

\subsection{Rationale}

Since there is not enough telemetry available to transmit the full information for each individual event, the event data are binned onboard into arrays as a function of species, energy, and angle. These arrays are referred to as the 3-D distribution products. The subtraction of the background contamination is done for each of the 3-D distribution products. The corrected 3-D distribution products can then be used to calculate any of the science products (moments of the distribution function, energy spectra, etc.) in the usual manner.

Since a background rate measurement is not implemented in the instrument, the background rate is estimated using the counts from the lowest energy channel of the $\mathrm{O}^{+} 3$-D science data, under the assumption that during the radiation belt passes counts in this energy channel are all due to the penetrating relativistic electrons. One particular exception to this assumption is the presence of low energy field aligned $\mathrm{O}^{+}$ populations frequently observed in the inner magnetosphere. Hence an extra precaution is taken. The $180^{\circ}$ instrument field of view is divided into 8 instrument anodes. How these 8 anodes and the spacecraft spin phase are combined to give the angle for the 3-D products is shown in Fig. 8 of Kistler et al. (2013). The background rate is deduced not from the counts registered in all the instrument anodes but from the counts registered in the two equatorial anodes, 4 and 5 (out of 8), only. This is because during the CLUSTER radiation belt encounters, the magnetic field is generally oriented along (or close to) the instrument $z$ axis, in which case the two equato-

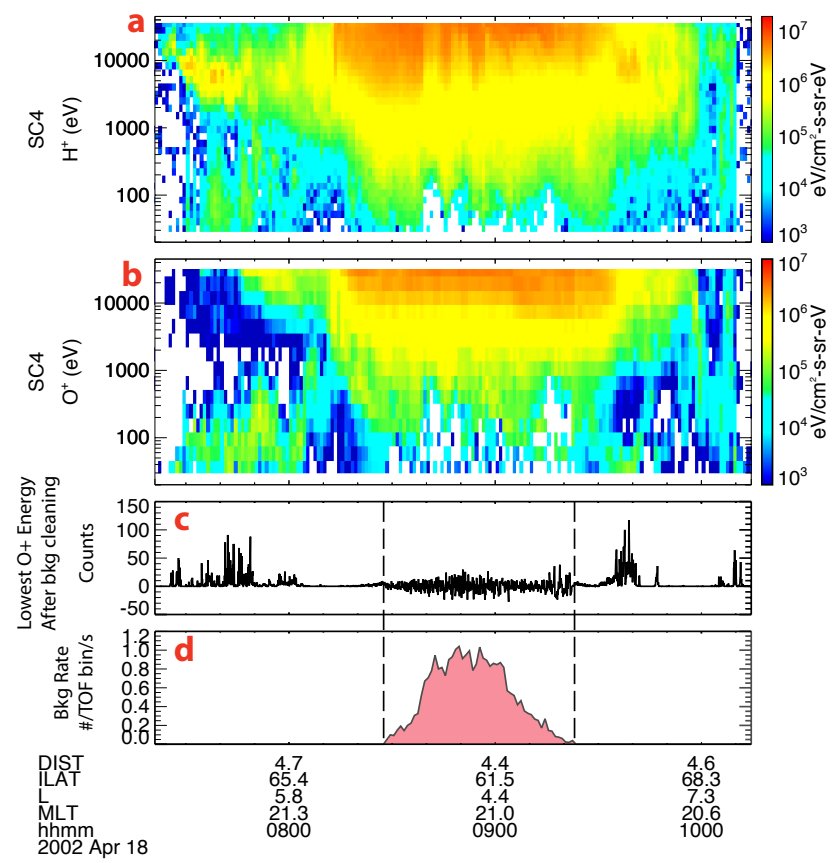

Fig. 3. Contamination subtracted $\mathrm{H}^{+}$(a) and $\mathrm{O}^{+}$(b) spectra. The dashed lines correspond to the interval that the background subtraction was applied. (c) shows counts in the lowest energy channel after the subtraction, which should average to zero. (d) shows the inferred background rate.

rial anodes are the ones that have the least chance to register counts from the field aligned populations.

\subsection{Implementation}

The implementation of this technique requires a number of steps. First, the exact time interval that the instrument registers background counts due to the radiation belt encounter has to be identified. Low energy $\mathrm{O}^{+}$populations are commonly observed during the inner magnetosphere passes and these intervals have to be distinguished from what is assumed to be background contamination. For example, such populations are evident in the $\mathrm{O}^{+}$energy spectra shown in Fig. 1 from 07:30 to 08:00 UT. The background interval selection is done visually using the $\mathrm{O}^{+}$energy spectra for each inner magnetosphere pass. The start and end times for each radiation belt encounter and for each spacecraft are stored in text files that are read by the background subtraction procedure. The dashed vertical lines in Fig. 3 indicate such an interval.

Next, the counts that are registered as $\mathrm{O}^{+}$in the lowest energy channel are converted into counts/second per TOF bin. This normalized background rate takes into account the number of TOF channels (bins) that correspond to the lowest energy channel $\mathrm{O}^{+}$and the integration time for this particular product. The background rate for the 18 April 2002 radiation belt encounter is shown in the bottom panel of Fig. 3. The top panel in Fig. 4 shows an example of the angular distribution 
of the background contaminated data of a 3-D $\mathrm{O}^{+}$distribution. Each "globe" corresponds to a single instrument energy channel. The top left globe corresponds to the highest energy channel, while the bottom right globe corresponds to the lowest energy and will be used as the background monitor.

Assuming that all TOF channels, for all energies, register the same number of background counts, the background rate can be used to reconstruct the background contamination for each energy channel and each species. However, as was mentioned earlier, the two equatorial anodes have the least chance to register counts from actual field-aligned populations. Therefore, the average of the normalized counts deduced from anodes 4 and 5 of the lowest energy $\mathrm{O}^{+}$channel will be used as the background rate instead. In order to convert this background rate into the background that the rest of the anodes would observe, the cross-anode efficiencies of the instrument for the radiation belt electrons are required. In Sect. 3.4, detailed information on how this is done, is provided.

From the deduced normalized background rate the background 3-D distributions can be reconstructed, for each species, for the whole time interval of the radiation belt encounter. Depending on the species that is being cleaned, the appropriate background 3-D distribution is reconstructed. First the background rate, determined from the $\mathrm{O}^{+}$, is interpolated to correspond with the time resolution for the particular species (different species/products can have different time resolution). Then, assuming that the background counts distribution is isotropic, the background counts are equally distributed on each anode over the corresponding angular bins (allowing for fractional counts). The middle panel in Fig. 4 shows the resulting background 3-D distribution for $\mathrm{O}^{+}$.

Finally, for each energy channel, the background distribution is subtracted from the original distribution (top panel) to produce the "cleaned" distribution (bottom panel). The resulting energy spectra are shown in Fig. $3 \mathrm{a}$ and $\mathrm{b}$ for $\mathrm{H}^{+}$ and $\mathrm{O}^{+}$respectively. It is important to note that the subtraction can result in negative counts at the angular bin level. Both the foreground and background counts are distributed in a Poisson distribution, so any count level $N$, has a statistical uncertainty of $\sqrt{N}$. Thus in regions that are background dominated, the subtraction will result in both positive and negative numbers due to the statistical variation. Those negative values are kept as is. Rejecting the negative values would "produce" artificial flux. It is important that a long time average reproduces the actual count rate for the particular interval. For example, this is shown in Fig. 3c where the "cleaned" counts for the lowest $\mathrm{O}^{+}$energy channel are plotted. The interval between the dashed lines was used to determine the background rate and by definition after the cleaning it should average to zero. A longer time average would show that indeed this is the case. In fact, we use this as a test against over/under subtraction.

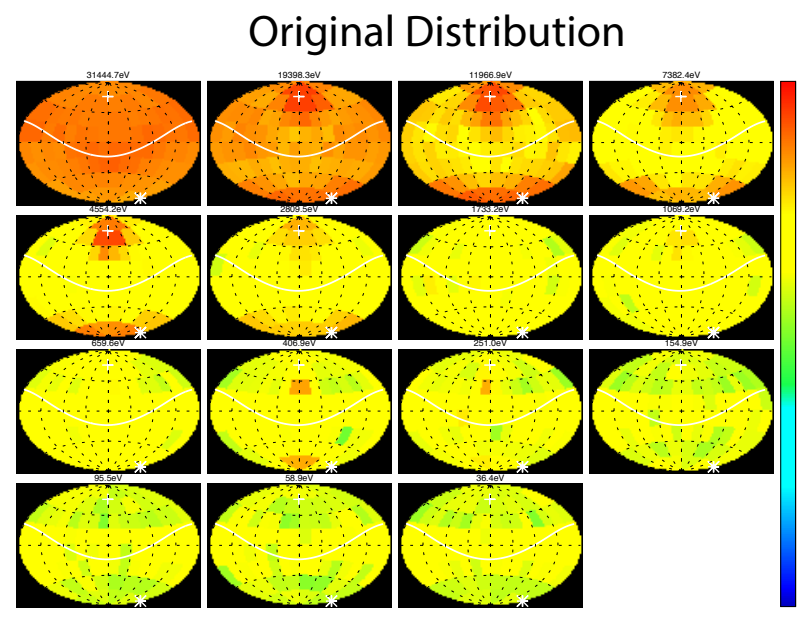

\section{Background Distribution}

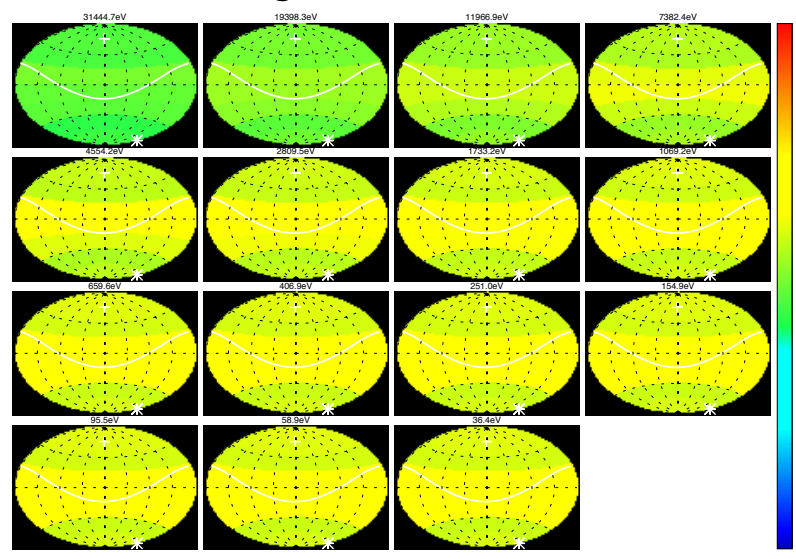

Cleaned Distribution

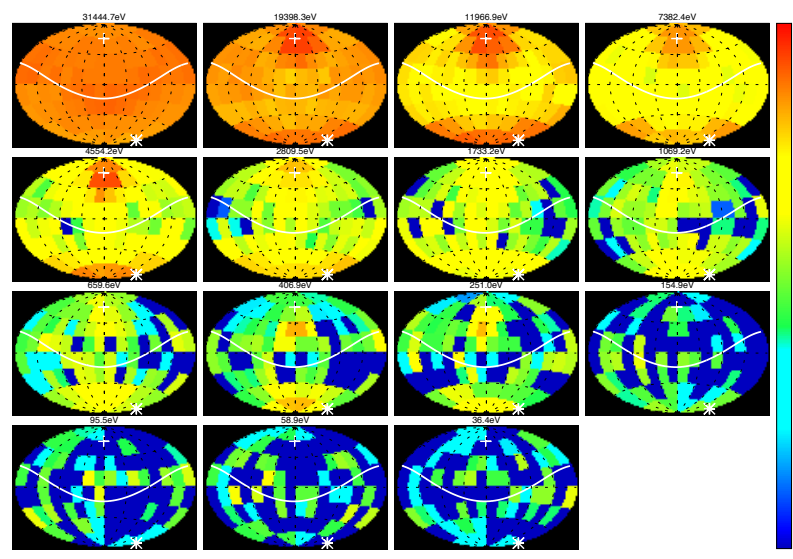

Fig. 4. Example of a contaminated $\mathrm{O}^{+}$distribution (top), the deduced background distribution (middle) and the resulted "cleaned" distribution (bottom). The individual "globes" correspond to the different energies with the top left globe corresponding to the highest energy channel and the bottom right globe corresponding to the lowest energy. Each globe shows the angular resolution in azimuthal and longitudinal directions. 


\subsection{Fine tuning the background rate}

When the background rate deduced from the $\mathrm{O}^{+}$window TOF bins is applied to the $\mathrm{H}^{+}$background subtraction it becomes apparent that the normalized counts for a particular radiation background level is not the same for all the TOF bins. Early TOF bins (closer to the $\mathrm{H}^{+}$range) have higher background counts compared to the $\mathrm{O}^{+}$TOF bins and therefore an adjustment is needed for $\mathrm{H}^{+}$and $\mathrm{He}^{+}$.

For this reason, the 3-D distribution of background counts for each species is multiplied by a certain species dependent factor. The factor for $\mathrm{O}^{+}$is 1 ( since $\mathrm{O}^{+}$is used to deduce the background level). For the other species, the factor was deduced by manually changing this factor and visually inspecting the cleaned energy spectrum. The variability of this factor was small and the values 1.65 and 1.2 were selected for $\mathrm{H}^{+}$and $\mathrm{He}^{+}$, respectively.

Subsequently, these factors were tested for selected events where it was evident (from visual inspection of the energy spectrograms) that the observed $\mathrm{H}^{+}$or $\mathrm{He}^{+}$counts at the lowest energy channels were only due to background. The requirement then was that after the background subtraction the average counts of the lowest energy channel are averaging to zero.

An additional step for the fine-tuning of the background subtraction is the use of the pitch angle spectra where background would appear as an isotropic population. Often, after the subtraction, strongly anisotropic populations are revealed that provide good reference points in deciding the exact level of background.

Finally, the "cleaned" counts are assigned an error bar based on the counting statistics for each time period that gives the statistical significance of the resulting fluxes. The relative error is calculated using

$\frac{\sqrt{C+C_{\mathrm{b}}}}{C-C_{\mathrm{b}}}$

where $C$ is the observed counts and $C_{\mathrm{b}}$ is the estimated background counts.

\subsection{Radiation belt electron cross-anode efficiencies}

The determination of the response of each anode of the CODIF instrument to the relativistic electron radiation is necessary. Although the anode efficiencies for each ion species is known from the instrument calibrations, the anode response to the penetrating radiation belt electrons is not necessarily the same. Therefore, this response has to be determined in order to be able to convert the counts registered in the equatorial anodes 4 and 5 into the counts that the rest of the anodes would register.

Figure 5 shows the normalized counts registered in each anode (anode counts over total counts) for the lowest $\mathrm{O}^{+}$energy channel during the radiation belt pass on 18 April 2002. All anodes show an interval where the normalized response

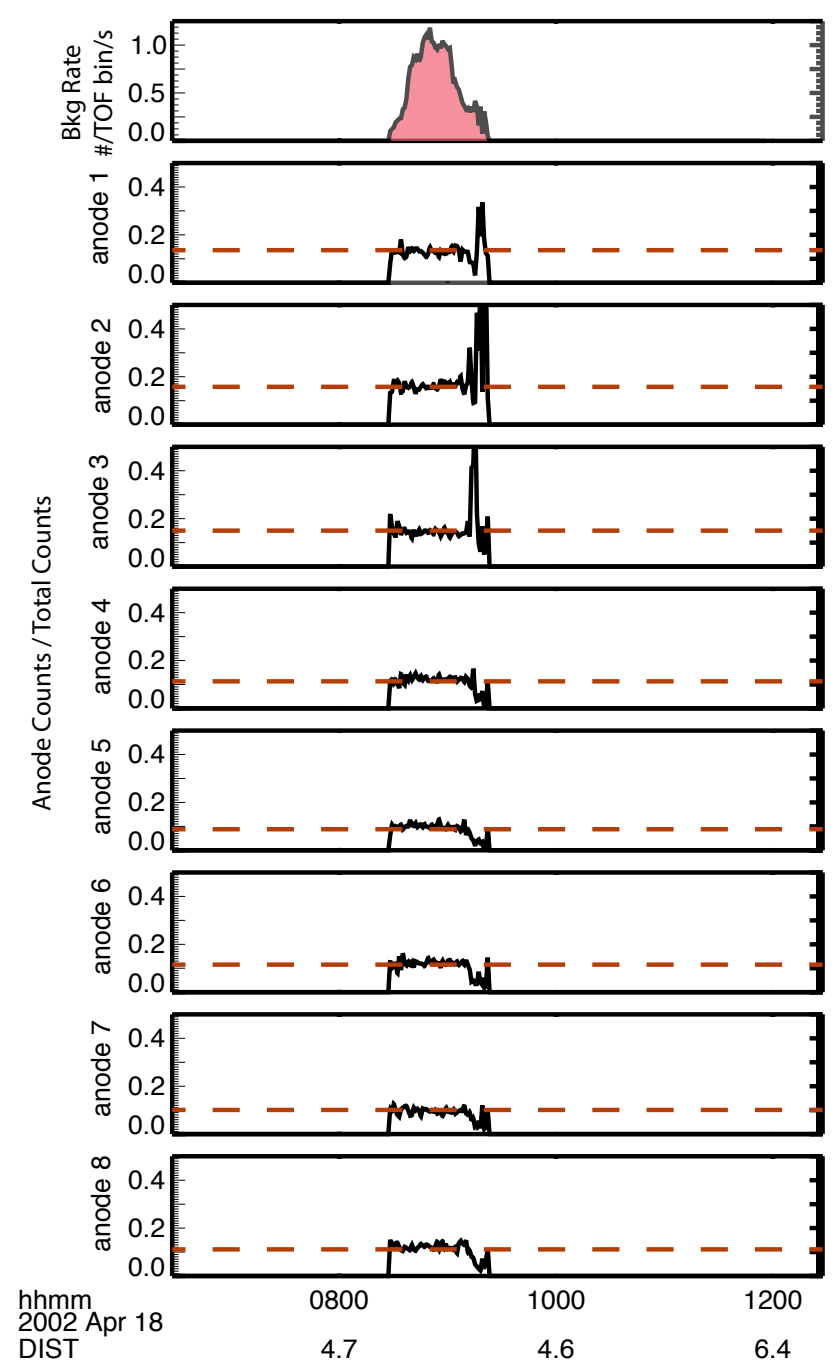

Fig. 5. Cross-anode efficiency determination of the penetrating electron response.

is flat, that is, all anodes detected the same profile. This is an indication that these counts are solely due to the penetrating electrons. The level of this flat response, indicated by the dashed red lines, is different for each anode and indicates the different response of each anode. These levels are used to determine the cross-anode efficiencies to the radiation belt electrons.

These cross-anode efficiencies are deduced from the data once per month for the mission's lifetime. If these efficiencies are not relatively accurate they will result in a non-zero (positive or negative) average of the counts of the "lowest" $\mathrm{O}^{+}$energy channel after the subtraction.

\section{$4 \mathrm{H}^{+}$spillover subtraction from the $\mathrm{He}^{+}$channel}

Figure 6 shows the CODIF TOF spectrum for all energies from the pulse-height (PHA) data collected from the plasma 


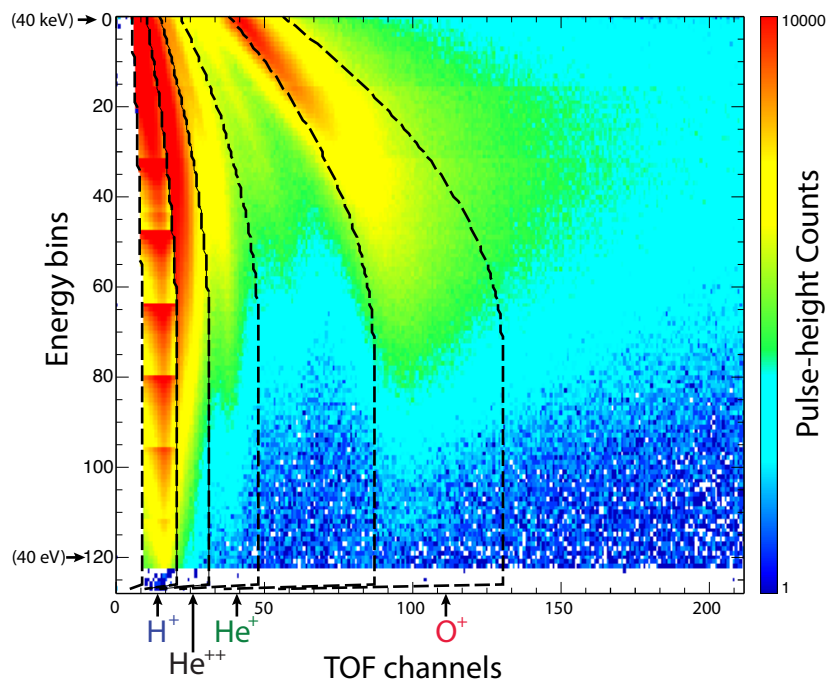

Fig. 6. CODIF time-of-flight spectrum for all energies. The dashed lines indicate the TOF windows for the four species, $\mathrm{H}^{+}, \mathrm{He}^{++}$, $\mathrm{H}^{+}$and $\mathrm{O}^{+}$.

sheet during the Cluster 2002 "tail season". The pulse-height data give the full information recorded by the instrument for a small sample of individual ion events. The PHA data are a low priority product, have a very coarse time resolution and are transmitted mainly for diagnostic purposes. However, they provide the full TOF channel vs. energy information allowing the reconstruction of the TOF distribution of the ion counts for each species and energy within the sample. The dashed lines, in Fig. 6, indicate the TOF windows used to characterize the different species in the transmitted science data. The $\mathrm{H}^{+}$counts in these pulse-height data are undersampled in order to get a good sample of the heavy ion data.

In Sect. 2 it was shown that although $\mathrm{H}^{+}, \mathrm{He}^{+}$and $\mathrm{O}^{+}$ are clearly separated in the TOF spectrum, the $\mathrm{He}^{++}$is in the tail of the $\mathrm{H}^{+}$distribution. This is also clearly seen in Fig. 6, where the high count rates of the $\mathrm{H}^{+}$"spill" into the $\mathrm{He}^{++}$window. However, a very small percentage of the $\mathrm{H}^{+}$ counts "spills" into the $\mathrm{He}^{+}$TOF window as well. Although the fraction of the $\mathrm{H}^{+}$that "spills" into the $\mathrm{He}^{+}$is small ( $\sim 0.3-1.7 \%$ according to Table 1$)$, the amount of $\mathrm{He}^{+}$relative to $\mathrm{H}^{+}$can also be very small, particularly during solar minimum, resulting in significant contamination. In order to be able to accurately track the $\mathrm{He}^{+}$as a function of the solar cycle, we have implemented an algorithm that corrects this spillover. It should be noted that there are also many time periods, particularly in the inner magnetosphere, where the $\mathrm{He}^{+}$flux is significant, and even exceeds the $\mathrm{H}^{+}$flux at some energies. In these cases the $\mathrm{H}^{+}$spillover is not an issue.

In order to be able to correct this spillover, we need to know what percentage of the $\mathrm{H}^{+}$counts spills into the $\mathrm{He}^{+}$ TOF window as a function of energy. This is addressed using long time averages from plasma-sheet measurements in the

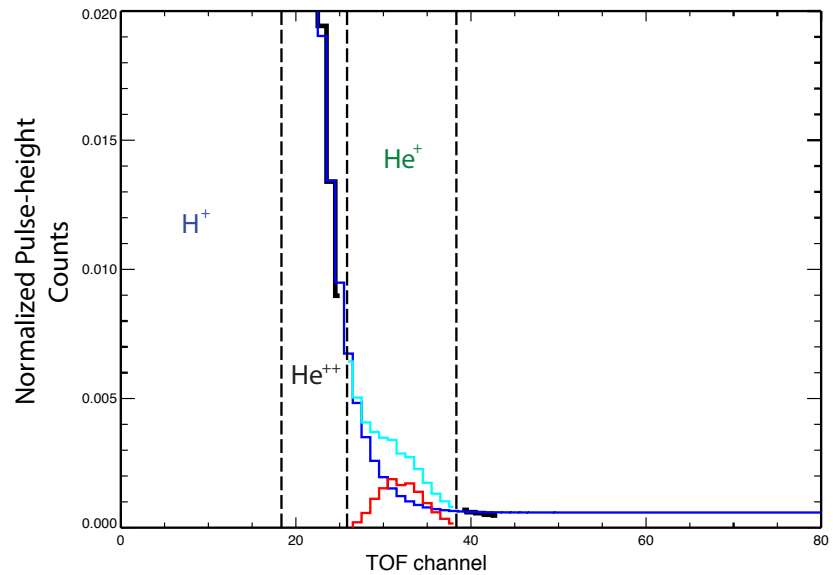

Fig. 7. Example of time-of-flight spectrum from a plasma-sheet period. The dashed lines show the TOF windows for $\mathrm{H}^{+}, \mathrm{He}^{++}$and $\mathrm{He}^{+}$. The black line together with the light blue line show the actual data. The light blue line indicates the registered counts in the $\mathrm{He}^{+}$TOF window. The blue line shows the fit to the two black line segments. The red line shows the subtracted counts in the $\mathrm{He}^{+}$window.

magnetotail, under the assumption that in the plasma sheet all the observed $\mathrm{He}^{++}$is due to spillover from the $\mathrm{H}^{+}$.

TOF spectra, as shown in Fig. 6, from the plasma sheet are accumulated for each year between 2001 and 2007. Such a TOF spectrum for one energy channel is shown in Fig. 7. Here, only a part of the TOF spectrum is shown. First the PHA data from the $\mathrm{He}^{++}$TOF window and from a small TOF interval after the $\mathrm{He}^{+}$TOF window, indicated by the two black line segments, are fitted with an exponential function $\left(y=\mathrm{ae}^{\left(-x^{d} / p\right)}+c\right)$. The resulting fit (dark blue line) provides the estimated tail of the $\mathrm{H}^{+}$TOF window distribution and the part of it that falls within the $\mathrm{He}^{+}$window is the estimated $\mathrm{He}^{+}$contamination. Next, the estimated contamination is subtracted from the $\mathrm{He}^{+}$TOF data (light blue), which results in the cleaned $\mathrm{He}^{+}$data (red line). The ratio of the estimated $\mathrm{He}^{+}$contamination counts over the registered $\mathrm{He}^{++}$ counts provides the percentage of $\mathrm{He}^{++}$that spills in the $\mathrm{He}^{+}$ TOF window. This percentage is calculated for each energy bin and for each year (using plasma-sheet data only) and is shown in Fig. 8a.

Next, again under the assumption that all the $\mathrm{He}^{++}$observed is $\mathrm{H}^{+}$spillover, we use the science data to determine the $\mathrm{H}^{+}$percentage that spills in the $\mathrm{He}^{++}$. Once more, this is done for each energy bin and for each year. The resulting ratios, shown in Fig. 8b, are the median values. The energy dependence for both ratios is the result of the dependence of the species TOF peak position on the particle energy. The width of the $\mathrm{H}^{+}$peak is dominated by electronic effects, and does not vary significantly with energy. Therefore, as the TOF peaks move closer together at higher energies (lower energy bins), there is more spillover. Finally, the product of 

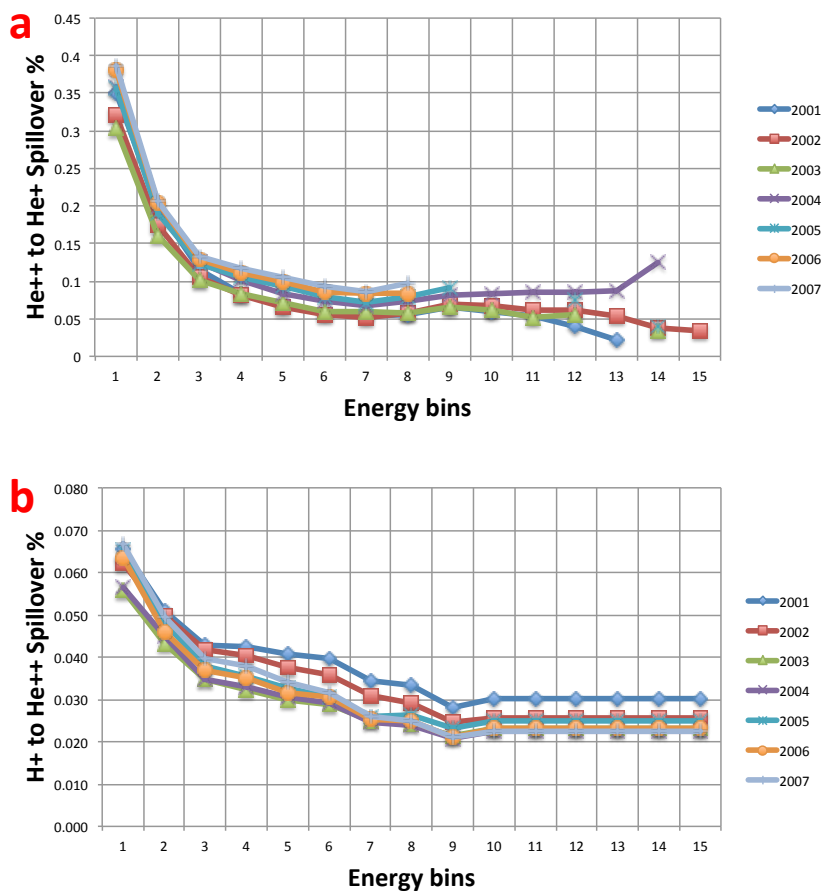

Fig. 8. (a) The $\mathrm{He}^{++}$percentage that spills in the $\mathrm{He}^{+}$as a function of energy for the period 2001-2007. (b) The $\mathrm{H}^{+}$percentage that spills in the $\mathrm{He}^{++}$as a function of energy for the same period. The percentage of the $\mathrm{H}^{+}$counts that spill into the $\mathrm{He}^{+}$is the product of the two percentage factors.

these two ratios provides the percentage of $\mathrm{H}^{+}$that spills in the $\mathrm{He}^{+}$TOF window.

It is important to reiterate that the cleaned data set will have a higher statistical error because the accuracy of the density estimate depends strongly on the level of the background subtracted. At this point the spillover subtraction has been applied for the density moment. While it is possible to calculate the higher moments, the main point of this product is to extract $\mathrm{He}^{+}$when statistics are very low, so we are still testing whether valid higher order moments can be obtained in these situations. However, as always, data analysis must be done intelligently, not blindly. A good check for whether the original data can be used would be to check the agreement between the "corrected" density and the "original" density. If agreement is good, then the subtraction is small and the rest of the moments should be valid.

\section{Summary}

We have presented two methods of background subtraction for the Cluster/CODIF instrument. The first method applies to background contamination due to penetrating electron radiation commonly occurring during the encounters with the Earth's radiation belts and affects all species. Although TOF spectrometers, like CODIF, are relatively immune to back- ground contamination due to the double coincidence requirement, high background rates can still result in false coincidences. A second type of background in these instruments occurs when events of one species, $\mathrm{H}^{+}$, fall into the time-offlight range defined for another species, $\mathrm{He}^{+}$. Although the fraction of the $\mathrm{H}^{+}$events that spill into the $\mathrm{He}^{+}$measurement is small, when the actual $\mathrm{He}^{+}$fluxes are low this can result in significant contamination. Both techniques work well; however, when the signal-to-background ratio becomes small the statistical error of the resulting "cleaned" measurements is correspondingly large.

Acknowledgements. We are grateful to the many engineers and scientists from UNH, MPE, CESR, MPS, IFSI, IRF, UCB and UW who made the development of the CIS instrument possible. This work at UNH was supported by NASA under grant NNX11AB65G.

Edited by: H. Laakso

\section{References}

Escoubet, C. P., Fehringer, M., and Goldstein, M.: Introduction The Cluster mission, Ann. Geophys., 19, 1197-1200, doi:10.5194/angeo-19-1197-2001, 2001.

Kistler, L. M., Klecker, B., Jordanova, V. K., Möbius, E., Popecki, M. A., Patel, D., Sauvaud, J. A., Rème, H., Di Lellis, A. M., Korth, A., McCarthy, M., Cerulli, R., Bavassano Cattaneo, M. B., Eliasson, L., Carlson, C. W., Parks, G. K., Paschmann, G., Baumjohann, W., and Haerendel, G.: Testing electric field models using ring current ion energy spectra from the Equator-S ion composition (ESIC) instrument, Ann. Geophys., 17, 1611-1621, doi:10.1007/s00585-999-1611-2, 1999.

Kistler, L. M., Mouikis, C. G., and Genestreti, K. J.: In-flight calibration of the Cluster/CODIF sensor, Geosci. Instrum. Method. Data Syst., 2, 225-235, doi:10.5194/gi-2-225-2013, 2013.

Möbius, E., Kistler, L. M., Popecki, M. A., Crocker, K. C., Granoff, M., Jiang, Y., Sartori, E., Rème, H., Sauvaud, J.A., Cros, A., Aoustin, C., Camus, T., Medale, J.-L., Rouzaud, J., Carlson, C. W., McFadden, J. P., Curtis, D., Heetderks, H., Croyle, J., Ingraham, C., Klecker, B., Hovestadt, D., Ertl, M., Eberl, F., Kastle, H., Kunneth, E., Laeverenz, P., Seidenschwang, E., Shelley, E. G., Klumpar, D. M., Hertzberg, E., Parks, G. K., McCarthy, M., Korth, A., Rosenbauer, H., Grave, B., Eliasson, L., Olsen, S., Balsiger, H., Schwab, U., and Steinacher, M.: The 3-D Plasma Distribution Function Analyzers With Time-of-Flight Mass Discrimination for Cluster, FAST, and Equator-S, in: Measurement Techniques in Space Plasmas - Particles, edited by: Pfaff, R. F., AGU Geophysical Monograph, 102, 243-248, 1998.

Rème, H., Aoustin, C., Bosqued, J. M., Dandouras, I., Lavraud, B., Sauvaud, J. A., Barthe, A., Bouyssou, J., Camus, Th., Coeur-Joly, O., Cros, A., Cuvilo, J., Ducay, F., Garbarowitz, Y., Medale, J. L., Penou, E., Perrier, H., Romefort, D., Rouzaud, J., Vallat, C., Alcaydé, D., Jacquey, C., Mazelle, C., d'Uston, C., Möbius, E., Kistler, L. M., Crocker, K., Granoff, M., Mouikis, C., Popecki, M., Vosbury, M., Klecker, B., Hovestadt, D., Kucharek, H., Kuenneth, E., Paschmann, G., Scholer, M., Sckopke, N., Seidenschwang, E., Carlson, C. W., Curtis, D. W., Ingraham, C., Lin, R. 
P., McFadden, J. P., Parks, G. K., Phan, T., Formisano, V., Amata, E., Bavassano-Cattaneo, M. B., Baldetti, P., Bruno, R., Chionchio, G., Di Lellis, A., Marcucci, M. F., Pallocchia, G., Korth, A., Daly, P. W., Graeve, B., Rosenbauer, H., Vasyliunas, V., McCarthy, M., Wilber, M., Eliasson, L., Lundin, R., Olsen, S., Shelley, E. G., Fuselier, S., Ghielmetti, A. G., Lennartsson, W., Escoubet, C. P., Balsiger, H., Friedel, R., Cao, J.-B., Kovrazhkin, R. A., Papamastorakis, I., Pellat, R., Scudder, J., and Sonnerup, B.: First multispacecraft ion measurements in and near the Earth's magnetosphere with the identical Cluster ion spectrometry (CIS) experiment, Ann. Geophys., 19, 1303-1354, doi:10.5194/angeo19-1303-2001, 2001.
Wang, C.-P., Gkioulidou, M., Lyons, L. R., Wolf, R. A., Angelopoulos, V., Nagai, T., Weygand, J. M., and Lui, A. T. Y.: Spatial distributions of ions and electrons from the plasma sheet to the inner magnetosphere: Comparisons between THEMIS-Geotail statistical results and the Rice convection model, J. Geophys. Res., 116, A11216, doi:10.1029/2011JA016809, 2011.

Wüest, M.: Time-of-Flight Ion Composition Measurement Technique for Space Plasmas, in: Measurement Techniques in Space Plasmas - Particles, 102, edited by: Pfaff, R. F., AGU Geophysical Monograph, 102, p. 141-155, 1998. 